\title{
Wiring Up Vision: Minimizing Supervised Synaptic Updates Needed to Produce a Primate Ventral Stream
}

\author{
Franziska Geiger* ${ }^{*}, 1,2,3,4$, Martin Schrimpf ${ }^{*}, 1,5,6$, Tiago Marques ${ }^{1,5,6}$, and James J. DiCarlo ${ }^{1,5,6}$ \\ ${ }^{1}$ McGovern Institute for Brain Research, MIT \\ ${ }^{2}$ University of Augsburg \\ ${ }^{3}$ Ludwig Maximilian University \\ ${ }^{4}$ Technical University of Munich \\ ${ }^{5}$ Department of Brain and Cognitive Sciences, MIT \\ ${ }^{6}$ Center for Brains, Minds and Machines, MIT \\ *joint first authors
}

\begin{abstract}
After training on large datasets, certain deep neural networks are surprisingly good models of the neural mechanisms of adult primate visual object recognition. Nevertheless, these models are poor models of the development of the visual system because they posit millions of sequential, precisely coordinated synaptic updates, each based on a labeled image. While ongoing research is pursuing the use of unsupervised proxies for labels, we here explore a complementary strategy of reducing the required number of supervised synaptic updates to produce an adult-like ventral visual stream (as judged by the match to V1, V2, V4, IT, and behavior). Such models might require less precise machinery and energy expenditure to coordinate these updates and would thus move us closer to viable neuroscientific hypotheses about how the visual system wires itself up. Relative to the current leading model of the adult ventral stream, we here demonstrate that the total number of supervised weight updates can be substantially reduced using three complementary strategies: First, we find that only $2 \%$ of supervised updates (epochs and images) are needed to achieve $\sim 80 \%$ of the match to adult ventral stream. Second, by improving the random distribution of synaptic connectivity, we find that $54 \%$ of the brain match can already be achieved "at birth" (i.e. no training at all). Third, we find that, by training only 5\% of model synapses, we can still achieve nearly $80 \%$ of the match to the ventral stream. When these three strategies are applied in combination, we find that these new models achieve $80 \%$ of a fully trained model's match to the brain, while using two orders of magnitude fewer supervised synaptic updates. These results reflect first steps in modeling not just primate adult visual processing during inference, but also how the ventral visual stream might be "wired up" by evolution (a model's "birth" state) and by developmental learning (a model's updates based on visual experience).
\end{abstract}

\section{Introduction}

Particular artificial neural networks (ANNs) are the leading mechanistic models of visual processing in the primate visual ventral stream [1,2]. After training on large-scale datasets such as ImageNet [3] and updating weights with back-propagation in the process, internal representations of these ANNs partly match neural representations in the primate visual system from early visual cortex V1 through V2 and V4 to high-level IT [4-7, 1, 2], and patterns of model object recognition behavior can partly account for patterns of primate object recognition behavior $[8,1,2]$. 
However, all the current top models of the primate ventral stream rely on trillions of supervised synaptic updates, i.e. the training of millions of parameters with millions of labeled examples over dozens of epochs. In biological systems on the other hand, the at-birth synaptic wiring as encoded by the genome already provides structure that is sufficient for squirrels to jump from tree to tree within months of birth, horses to walk within hours [9], and macaques to exhibit adult-like visual representations after months [10-12]. The heavy reliance of current ANNs on supervised synaptic updates has been a focus of critique in neuroscience; Zador [9] argues that "a child would need to ask one question every second of her life to receive a comparable volume of labeled data". While current models provide a basic understanding of the neural mechanisms of adult ventral stream inference, can we start to build models that provide an understanding of how the ventral stream "wires itself up" models of the initial state at birth and how it develops during postnatal life?

Related Work. Several papers have addressed related questions in machine learning: Distilled student networks can be trained on the outputs of a teacher network [13-15], and, in pruning studies, networks with knocked out synapses perform reasonably well [16, 17], demonstrating that models with many trained parameters can be compressed. Tian et al. [18] show that a pre-trained encoder's fixed features can be used to train a thin decoder with performance close to full fine-tuning and recent theoretically-driven work has found that training only BatchNorm layers [19] or picking the right parameters from a large pool of weights $[20,21]$ can already achieve high classification accuracy. Unsupervised approaches are also starting to develop useful representations without requiring many labels by inferring internal labels such as clusters or representational similarity [22-25]. Nevertheless, all of these approaches require many synaptic updates in the form of labeled samples or precise machinery to determine the right set of weights. In this work, we wanted to take first steps of using such models to explore hypotheses about the product of evolution (a model's "birth state") while simultaneously reducing the number of supervised synaptic updates (a model's visual experience dependent development) without sacrificing high brain predictivity.

Our contributions follow from a framework in which evolution endows the visual system with a well-chosen, yet still random "birth" pattern of synaptic connectivity (architecture + initialization), and developmental learning corresponds to training a fraction of the synaptic weights using very few supervised labels. Specifically,

1. we build models with a fraction of supervised updates (training epochs and labeled images) that retain high similarity to the primate ventral visual stream (referred to as brain predictivity),

2. we improve the "at-birth" synaptic connectivity to achieve reasonable brain predictivity with no training at all,

3. we propose a thin, "critical training" technique which reduces the number of trained synapses while maintaining high brain predictivity,

4. we combine these three techniques to build models with two orders of magnitude fewer supervised synaptic updates but high brain predictivity relative to a fully trained model

Code and pre-trained models will be available through GitHub.

\section{Modeling Primate Vision}

We evaluate all models on a suite of ventral stream benchmarks in Brain-Score [1], and we base the new models presented here on the CORnet-S architecture as this is currently the most accurate model of adult primate visual processing [2].

Brain-Score benchmarks. To obtain quantified scores for brain-likeness, we use a thorough set of benchmarks from Brain-Score [1]. All these benchmarks feed the same images to a candidate model that were used for primate experiments while "recording" activations or measuring behavioral outputs. Specifically, the V1 and V2 benchmarks present 315 images of 4deg naturalistic textures and compare model representations to primate single-unit recordings from Freeman et al. [26] (102 V1 and $103 \mathrm{~V} 2$ neurons); the V4 and IT benchmarks present 2,560 naturalistic 8deg images and compare models to primate Utah array recordings from Majaj et al. [27] (88 V4 and 168 IT electrodes). A linear regression is fit from model to primate representations in response to $90 \%$ of the images and its prediction score on the held-out $10 \%$ of images is evaluated with Pearson correlation, cross-validated 10 times. The behavioral benchmark presents 240 images of $8 \mathrm{deg}$ and compares model to primate 

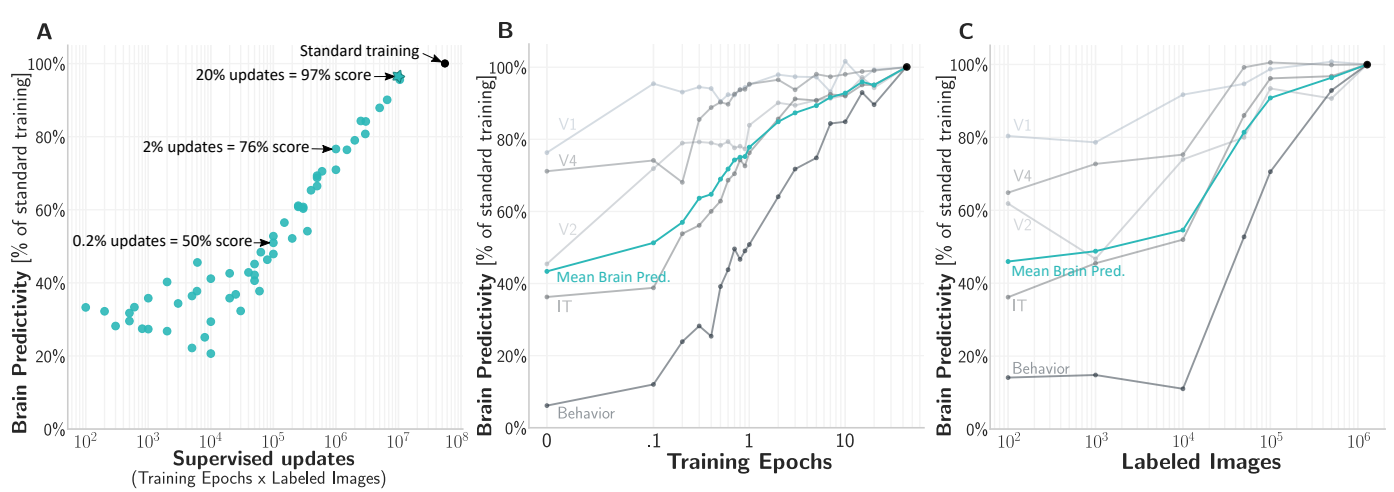

Figure 1: High brain predictivity can be achieved with few supervised updates ( $\log x$-axes). A Average brain predictivity of models trained with a range of supervised updates (epochs $\times$ images). Fairly brain-like representations are already realized with few supervised updates, relative to a fully trained model (black dot). B Individual brain predictivity scores over epochs. Models start to approximate the primate ventral stream with few training epochs. Lower visual areas (V1, V2) are approximated earlier in training. C Like B, but number of training images instead of epochs. Few images are sufficient to approximate especially early visual areas.

behavioral responses from Rajalingham et al. [8]. A logistic classifier is fit on models' penultimate representations on a separate set of 2,160 labeled images. The classifier is then used to estimate probabilities for 240 held-out images. Per-image confusion patterns between model and primate are compared with a Pearson correlation. All benchmark scores are normalized by the respective ceiling. We primarily report the average score as the mean of V1, V2, V4, IT, and behavioral scores.

Brain-Score provides separate sets of data as public benchmarks which we use to determine the type of distribution in Section 4, and the layer-to-region commitments of reference models.

CORnet-S. The current best model on the Brain-Score benchmarks is CORnet-S [2], a shallow recurrent model which anatomically commits to ventral stream regions. CORnet-S has four computational areas, analogous to the ventral visual areas V1, V2, V4, and IT, and a linear decoder that maps from neurons in the model's last visual area to its behavioral choices. The recurrent circuitry (Figure 3B) uses up- and down-sampling convolutions to process features and is identical in each of the models visual areas (except for $\mathrm{V} 1_{C O R}$ ), but varies by the total number of neurons in each area.

We base all models developed here on the CORnet-S architecture and use the same hyper-parameters as proposed in [2]. Representations are read out at the end of anatomically corresponding areas.

\section{High brain predictivity can be achieved with few supervised updates}

We evaluated the brain predictivity of CORnet-S variants that were trained with fewer epochs and images. Models are trained with an initial learning rate of 0.1 , divided by 10 when loss did not improve over 3 epochs, and stopping after three decrements.

Figure 1 shows model scores on neural and behavioral Brain-Score measures, relative to a model trained for 43 epochs on all 1.28M labeled ImageNet images. In Panel A, we compare the average score over the five brain measures of various models to the number of supervised updates that each model was trained with, defined as the number of labeled images times the number of epochs. While a fully trained model reaches an average score of .42 after 55,040,000 supervised updates ( 43 epochs $\times$ $1.28 \mathrm{M}$ images), a model with only 100,000 updates already achieves $50 \%$ of that score, and 1,000,000 updates increase brain predictivity to $76 \%$. Models are close to convergence score after 10,000,000 supervised updates with performance nearly equal to full training (97\%). Scores grow logarithmically with an approximate 5\% score increase for every order of magnitude more supervised updates.

Figures $1 \mathrm{~B}$ and $\mathrm{C}$ show individual neural and behavioral scores of models trained with fewer training epochs or labeled images independently. Early to mid visual representations (V1, V2, and V4 scores) seem to be especially closely met with only few supervised updates, reaching $50 \%$ of the final trained 
A

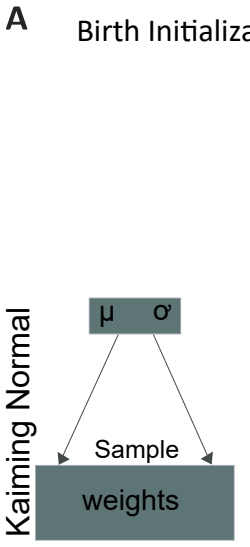

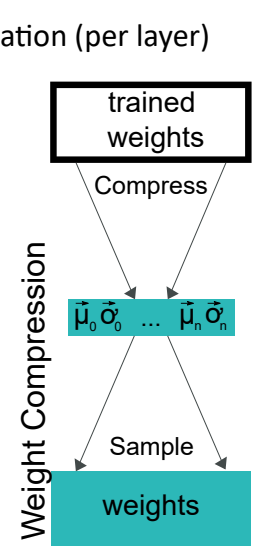

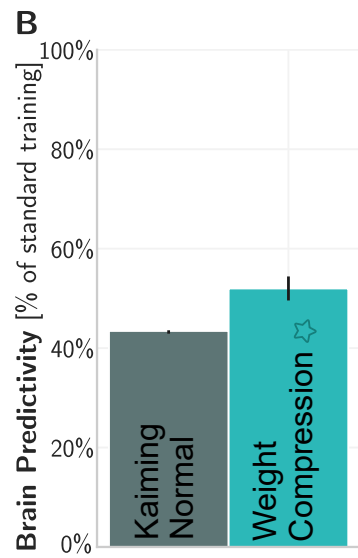

Figure 2: "At-birth" synaptic connectivity yields reasonable brain predictivity. A Specifying the initial weight distribution: Kaiming Normal $(K N)$ [28] samples from a generic Gaussian. Weight Compression (WC) compresses trained weights into lowparameter clustered distributions that weights can later be initialized from. B "At-birth" representations with $W C$ achieve 54\% brain predictivity of a fully trained model, with no training at all. Error bars represent SD.

model in fractions of the first epoch (Figure 1B). After only one full iteration over the training set, $\mathrm{V} 1, \mathrm{~V} 2$, and V4 scores are close to their final score (all $>80 \%$ ) while IT requires two epochs to reach a comparable level. Behavioral scores take slightly longer to converge ( $>80 \%$ after 7 epochs).

Similarly, when training until convergence with fractions of the $1.28 \mathrm{M}$ total images, 50,000 images are sufficient to obtain high neural scores ( $80 \%$ of full training in V1, V2, V4, IT). Behavioral scores again require more training: half the standard number of labeled images is needed to surpass $80 \%$.

Concretely relating supervised updates to primate ventral stream development, Seibert [12] establishes that no more than $\sim 4$ months - or 10 million seconds - of waking visual experience is needed to reach adult-level primate inferior-temporal cortex (IT; as assessed by its capability to support adult level object recogntion). From this estimate, we can compute how many supervised updates per second different models in Figure 1A would require (assuming those updates are evenly distributed over the 10 million seconds). For instance, the fully trained model's 55 million supervised updates translate to 5.5 updates every second, whereas the model with 1 million updates and $76 \%$ relative brain predictivity translates to one labeled image update every 10 seconds.

\section{4 "At-birth" synaptic connectivity yields reasonable brain predictivity with no training at all}

If few supervised updates can get model representations fairly close to a fully trained model (Figure 1), how close are the initial representations without any training? In relation to biology and following the introduced framework of treating all consecutive training as developmental learning, these "at-birth" synaptic connections would result from information encoded in the genome as a product of evolution.

Due to the genome's capacity bottleneck, it is thought to be infeasible to precisely encode every synapse. Primary visual cortex alone contains $\sim 1.4 \mathrm{E} 8$ neurons per hemisphere [29], $\sim 1 \mathrm{E} 3$ synapses per neuron, each requiring $\sim 35$ bits per synapse [9]. Thus, without any clever rules, specifying the connections in one hemisphere of $\mathrm{V} 1$ could require up to $\sim 4.9 \mathrm{E} 12$ bits - an order of magnitude more than the entire genome's $1 \mathrm{~GB}=8 \mathrm{E} 9$ bits [9].

Sampling synaptic weights from reasonably compressed distributions on the other hand places only little memory requirements on genetic encoding while potentially yielding reasonably useful initial weights. Seibert [12] for instance found that the representations in juvenile (19-32 weeks) primate high-level ventral stream IT seem no different from adult monkeys, suggesting that synaptic weights up to IT after that age change only minimally, if at all. Current machine learning techniques for initializing weights, such as Kaiming Normal [28], sample from a Gaussian distribution with $\mu=0$ and $\sigma=\sqrt{2} / N$ where $N$ is the number of incoming connections per layer.

To improve on Kaiming Normal initialization, we explored multi-dimensional distributions as a more expressive alternative. Like current initializations, these distributions only require a small number of parameters, but we explicitly specify them for each layer. To determine the right parameterization, we compress a trained model's weights into clusters which we then sample from ("Weight Compression"). 
More specifically, for all convolutional layers except the first, we cluster kernel weights in a layer using the k-nearest-neighbors algorithm [30]. The number of clusters is determined using elbow [31]. To capture the relative importance of clusters we fit a normal distribution $\mathcal{N}_{f}$ for each cluster with $\mu_{f}$ as the cluster frequency over kernels and $\sigma_{f}$ as the frequency standard deviation. To sample weights for a kernel, we first sample a cluster distribution $i \sim \mathcal{N}_{f}$ per kernel and then obtain channel weights by sampling from a Gaussian with $\vec{\mu}_{i}$ as the cluster center and the standard deviation $\vec{\sigma}_{i}$ of clustered weights. In batch normalization layers, we fit one normal distribution each to the weights and biases.

For the first convolutional layer only, we employ a Gabor prior on the weights following studies in V1 [32,33] by fitting channels' weights to a Gabor function and then fit a mixture-of-Gaussians to the Gabor parameters per kernel (supplement). To sample new weights, we sample Gabor parameters and set the weights to the thereby specified Gabor. Such a wiring mechanism might require more machinery than the direct distributional sampling employed in later layers - however, smooth Gabors could be implemented as a changing growth factor gradually modulating spatial connections [34].

Applying $W C$ to CORnet-S, we first obtain a compressed and clustered set of parameters, from which we sample entirely new weights to yield a new model CORnet- $\mathrm{S}_{\mathrm{WC}}$. This model is not trained at all and we only evaluate the goodness of its initial wiring on the suite of Brain-Score benchmarks. Strikingly, we find that even without any training, CORnet- $\mathrm{S}_{\mathrm{WC}}$ achieves $54 \%$ of the brain predictivity relative to a fully-trained model (Figure 2). Early ventral stream regions V1 and V2 are predicted especially well with no loss in score but we note that these two benchmarks are less well predicted by the trained model to begin with. V4 scores also approximate those of a trained model relatively well (75\%). The major drop occurs in the IT and especially behavioral scores where CORnet- $\mathrm{S}_{\mathrm{WC}}$ only reaches 39\% and 6\% of the trained model's score respectively. Similarly, a trained linear decoder on CORnet- $\mathrm{S}_{\mathrm{WC}}$ ' IT representations only reaches $5 \%$ of a trained model's ImageNet top- 1 accuracy.

\section{Training thin down-sampling layers reduces the number of updated synapses while maintaining high brain predictivity}

While improved "at-birth" synaptic connectivity can reach 54\% of a fully-trained model's score (Section 4), additional visual-experience dependent updates appear necessary to reach higher predictivities. With standard back-propagation, each such iteration updates millions of synaptic weights in the model, which, related to biology, would require precise machinery to coordinate these updates.

We propose a novel thin training technique which we term Critical Training (CT; Figure 3A). Instead of updating every single model synapse, $C T$ updates only the weights in down-sampling layers. In CORnet-S, each of the V2, V4, and IT blocks has one down-sampling layer to produce an area's final representation. We explore successive variants of applying $C T$ up to a block in the architecture and then training the following blocks, e.g. freezing V1, V2, V4 with critical training of the respective down-sampling layers and additional IT training. The final $C T$ ventral stream model is almost completely frozen and only the synapses generating each cortical area's output are trained.

We compared Critical Training to a naive approach of reducing the trained parameters by freezing model blocks from the bottom up, for instance keeping the V1 and V2 blocks fixed while training V4 and IT blocks. We term this block-wise freezing and training approach Downstream Training (DT).

Compared to standard back-propagation training all the weights, both CT and DT reduce the number of trained parameters (Figure 3B). However, while the average score with $D T$ (gray) already drops below $65 \%$ with over a quarter of trained parameters remaining, $C T$ (blue) maintains over $75 \%$ with only 1.4 out of 52.8 million parameters trained. Note that we count model parameters and do not compute how many biological synapses each convolutional weight would be equivalent to. In detail, $C T$ maintains over $75 \%$ of the score in V1, V2, V4, IT, 58\% of behavior and 40\% ImageNet accuracy.

By reducing the number of trained parameters, Critical Training also yields engineering benefits in training time with a $30 \%$ reduction in the time per epoch at over $80 \%$ of the brain predictivity and more than $40 \%$ of the ImageNet score. The training time reduction is less drastic than the parameter reduction because most gradients are still computed for early down-sampling layers (Discussion). 
A



B



Frozen Trained

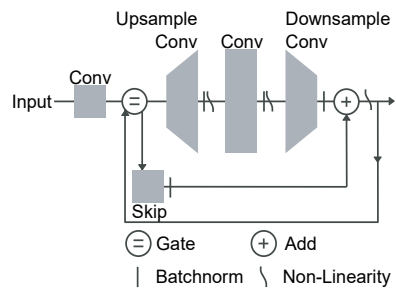

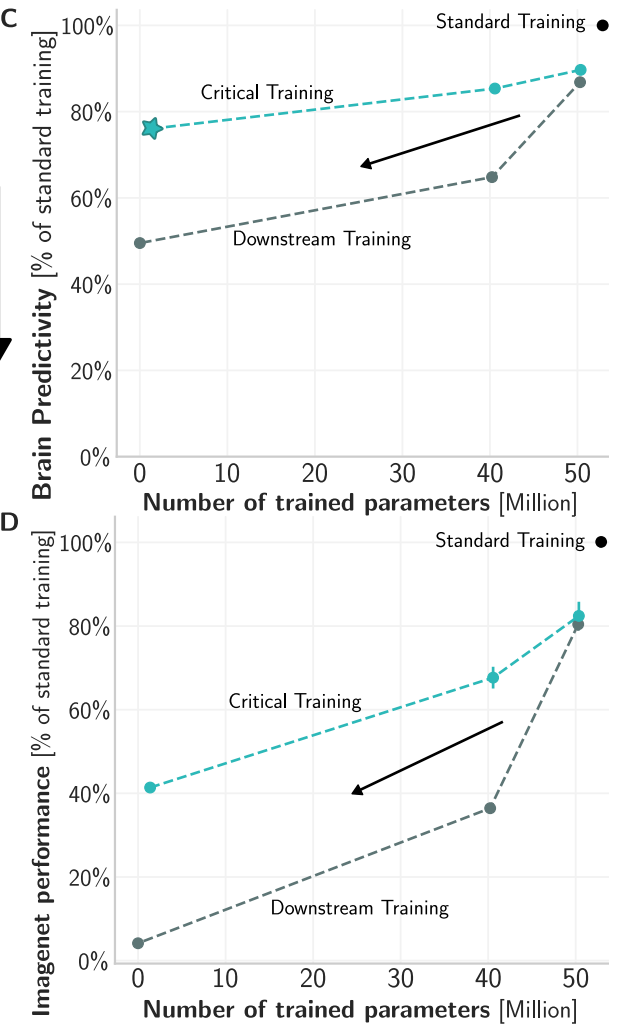

Figure 3: Training thin down-sampling layers reduces the number of updated synapses while maintaining high brain predictivity. A We could naively reduce the parameters of a fully-trained model by freezing layers from the bottom up, training only the top layers ("Downstream Training DT"; gray box). We instead propose Critical Training $(C T)$ which only trains down-sampling layers (blue box). B CORnet-S circuitry. $C T$ only trains the thin down-sampling convolution. $\mathbf{C}$ Naively reducing parameters from standard training (black dot, top right) quickly deteriorates brain predictivity ( $D T$, gray line) whereas Critical Training reduces parameters while retaining high scores (blue line, $C T$ ). D Like C, but measuring ImageNet score. $C T$ retains nearly half the score with a fraction of parameters.

\section{High brain predictivity can be achieved with a relatively small number of supervised synaptic updates}

All three training reduction methods independently minimize the number of supervised synaptic updates required to reach a reasonably high brain predictivity. Reducing the number of supervised updates minimizes required updates by a smaller number of epochs and images (Section 3); Weight Compression (WC) improves the at-birth synaptic connectivity for high initial scores with no training at all (Section 4); and Critical Training (CT) reduces the number of synapses that are updated during training (Section 5). We now combine these three methods to build novel models that only require a small number of supervised synaptic updates to reasonably capture the mechanisms of adult ventral visual stream processing and object recognition behavior.

Figure 4A shows the average brain predictivity of a range of models with varying numbers of supervised synaptic updates relative to a standard trained CORnet-S (black dot). With a reduced number of supervised updates (training epochs and labeled images) but standard initialization and training all weights (light blue dots), models require 5.2 trillion updates to achieve $>50 \%$ of the score of a fully trained model and about 100 trillion updates to reach $80 \%$ brain score. Adding $W C+C T$ (dark blue dots), the corresponding model already reaches 53\% at birth with 0 supervised synaptic updates. At $0.5 \%$ the updates of a fully trained model (14 trillion vs. 3000 trillion), models then reach $79 \%$ of the score (iz model with modeling choices marked in Figures 1 to 3). Reference models (gray dots) MobileNet [35] and ResNet [36] obtain high scores, but also require many supervised synaptic updates. HMAX [37] is fully specified with no updates but lacks in score. 

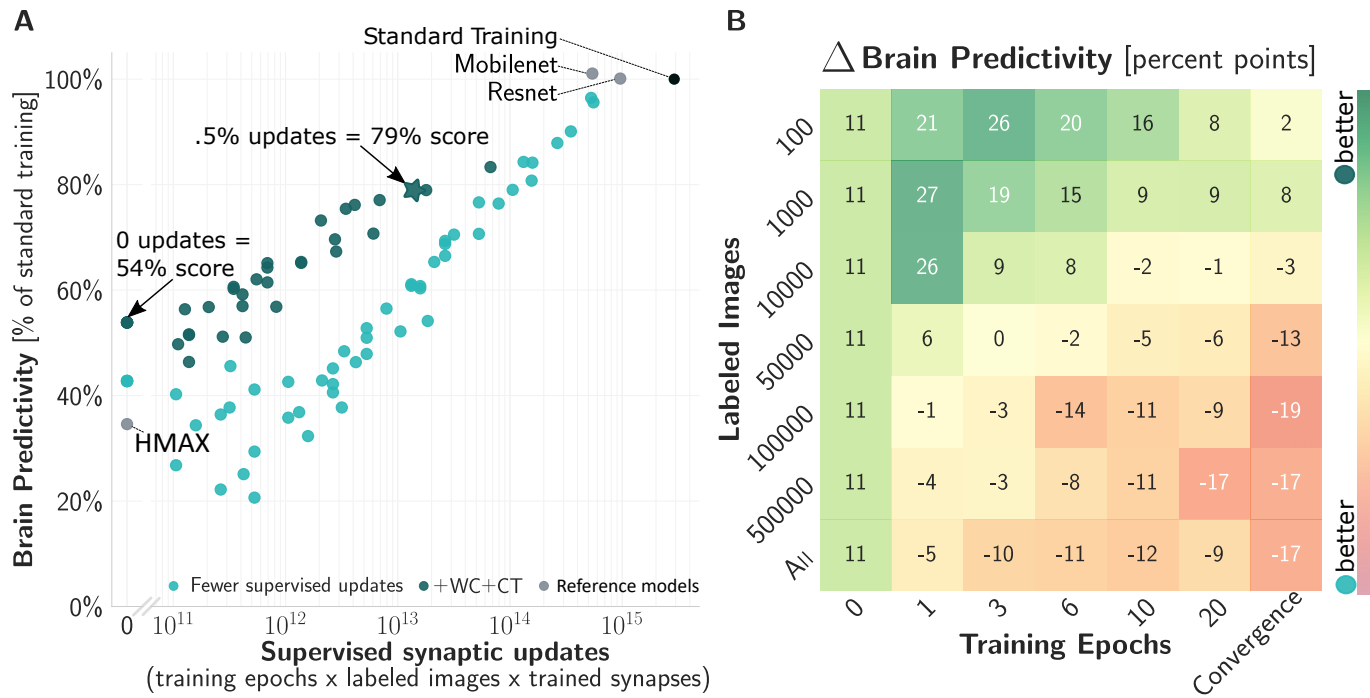

Figure 4: High brain predictivity can be achieved with few supervised synaptic updates through a combination of training reductions ( $\log x$-axis). A By reducing updates with a combination of fewer supervised updates (Figure 1), improved initialization WC (Figure 2), and training only downsampling layers $C T$ (Figure 3), the resulting models (dark blue dots; fewer supervised updates alone in light blue) maintain high brain predictivity while requiring only a fraction of supervised synaptic updates compared to standard CORnet-S (black dot, top right). B Comparison between $W C$-initialized models trained with $C T$ versus standardly initialized models training all weights, when varying training epochs and labeled images. Colors represent their percent point difference in brain predictivity. $W C+C T$ improve performance in regimes with few epochs and images.

Zooming in on individual benchmarks, early and mid visual cortex can be approximated by models that only require minimal to no training as well as a fully trained model: V1 reaches $>90 \%$ with no updates at all, V2 after 0.2 trillion updates, and V4 reaches $>80 \%$ after 18 trillion updates. Matching high-level visual cortex IT and behavioral outputs on the other hand requires more supervised synaptic updates, albeit still vastly fewer than often believed: with 68 trillion for $80 \%$ of IT and 35 trillion for $66 \%$ of behavior - all compared to a fully trained model's 3,000 trillion supervised synaptic updates.

We next examined interactions between the methods by comparing models initialized with $W C$ and trained with $C T$ to models with standard initialization and training all weights, when both are trained with fewer epochs and images. Figure 4B shows the percent point difference between the two model families. Positive numbers (green) indicate an improvement by using $W C+C T$ whereas negative numbers (red) indicate a decrease in score with respect to standard training. $W C+C T$ yield strong benefits in a regime with few supervised updates, improving by up to 27 percent points when training for only 1 epoch on 1,000 images. With many updates on the other hand, $W C+C T$ is actually less advantageous than standard training: with all 43 epochs and $1.28 \mathrm{M}$ images, the score reduces by 17 percent points. $W C+C T$ therefore most positively interacts with a small budget of supervised updates.

\section{Dissecting training reductions}

We asked whether the developed techniques would generalize to architectures other than the CORnet-S architecture that they were based on. We therefore applied Weight Compression (WC) and Critical Training (CT) to ResNet-50 [36] and MobileNet [35] architectures, both high-performing models on Brain-Score. We used WC distributions determined on CORnet-S, i.e. we tested their transfer without re-fitting. $W C+C T$ maintain most of the score in ResNet with $91 \%$ of the score despite an almost $80 \%$ reduction in parameters. When applied to MobileNet, the average score drops by $22 \%$ and parameters are reduced less strongly (43\%). This difference in retaining the score could be due to MobileNet already being very compressed, or having a less similar architecture.

With most analyses so far comparing an average score, we dissected the relative contributions of $W C$ and $C T$ to individual benchmarks (Figure 5B). We compared $K N$ to $W C$ initialization, as well as 

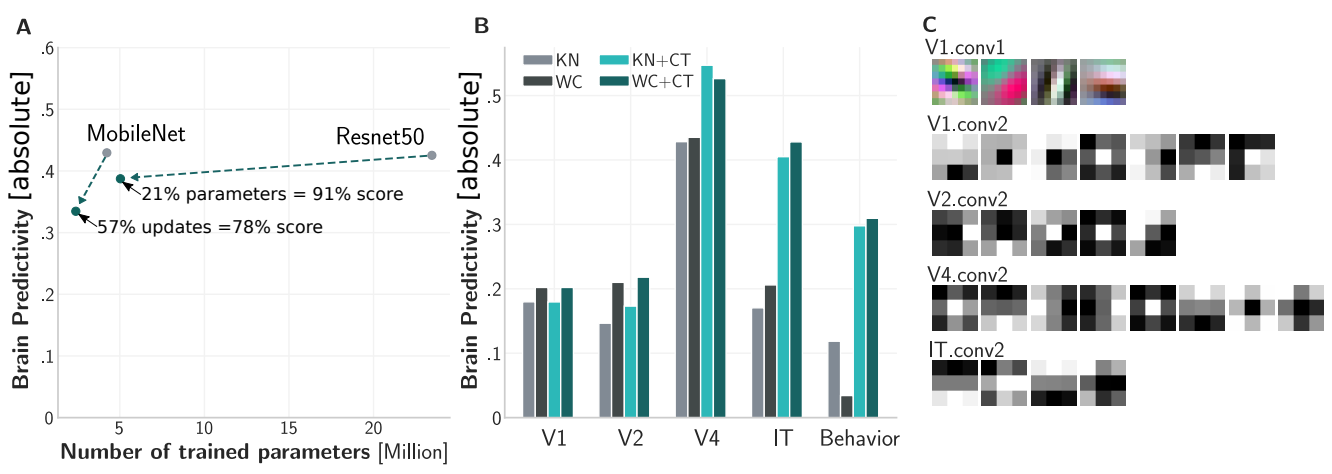

Figure 5: Dissecting training reductions. A Transfer to other networks. We sample from $W C$ initializations determined on CORnet-S, followed by Critical Training of only down-sampling layers. B Absolute scores on individual benchmarks of combinations of initialization $(K N / W C$, Figure 2), and with critical training ( $C T$, Figure 3 ) techniques. C Visualization of $W C$ kernel cluster centers.

resulting models after critical training $(K N+C T$ and $W C+C T)$. $W C$ initialization improves most over $K N$ in early visual regions $\mathrm{V} 1$ and V2, with additional gains in IT. Additional training with $C T$ is most beneficial in mid- to high-level visual cortex V4 and IT, as well as the behavioral benchmark.

Studies in model interpretability [38-40] analyze and classify model weights, similar to WC. Visualizing the centers of weight clusters at different locations in the network (Figure 5C), we find that the first layer's Gabors qualitatively align with an analysis by Cammarata et al. [40]. Consecutive cluster centers seem to represent an intuitive division of channel types with opposite types in every layer.

\section{Discussion}

We developed a range of models with neural and behavioral scores approaching those of the current leading model of the adult ventral visual stream, while requiring only a fraction of supervised synaptic updates. These models were built by complementarily 1) reducing the number of supervised updates, i.e. training epochs and labeled images; 2) improving the "at birth" distribution of synaptic connectivity; and 3) training only critical synapses at the end of each model area. The techniques and resulting models proposed here are first steps to more closely modeling not just adult primate visual processing, but also exploring the underlying mechanisms of evolution and developmental learning.

These first steps are far from accounting for the rich information encoded in the genome or the developmental learning that together result in adult mechanisms of visual processing. We here started from CORnet-S, which is the current leading model of the adult ventral stream, but does not fully predict neural or behavioral measurements. The architecture we based our techniques on might therefore be flawed. We verified favorable transfer to models with similar architectures such as ResNet, but generalization to an already compressed MobileNet was limited (Figure 5A).

Relating the proposed techniques to genomic mechanisms, such "principles" should generalize to other domains such as auditory processing. With the capacity bottleneck in the genome, mechanisms for wiring up would likely be shared between similar systems. With early visual areas being predicted much better than later ones by the model resulting from $W C$ initialization, early regions in general might be more hard-wired than later ones such that synaptic updates primarily take place in higher cortical regions based on representations hard-wired through DNA. One potential short-coming of $W C$ to account for higher regions is that it does not consider cross-layer dependencies, and incorporating these into mechanisms for wiring up might further improve representations without any training.

A critical component in more closely modeling primate development is to reduce the dependence on labels altogether. Recent unsupervised approaches are starting to rival the classification performance of supervised models [22-25] and combining them with the advances presented here could further reduce the number of synaptic updates. With critical training (Figure 3), only few weights need to be updated for high scores, so unsupervised learning might not need to tackle all the weights. Current unsupervised techniques still require back-propagation however which is routinely criticized as non- 
biological, among others due to the propagation of gradients [41-43]. Local learning rules might alleviate these concerns and additionally yield engineering gains due to increased parallelizability.

The changes to model initialization and training presented here already lead to models that more closely align with primate development than prior models, but they are still far from the actual biological mechanisms. We expect future work in this direction to further close the gap with improved evolutionarily encoded wiring mechanisms and developmental learning rules.

\section{Broader Impact}

The techniques proposed in this paper have broader implications for two fields:

First, the field of neuroscience may benefit from improved models of primate visual evolution, development, and function. These models may be useful in the eventual correction of diseases or abnormal development. However, excessive confidence in such systems may be equally dangerous and we here base "match-to-brain" on only a handful of measures. These models further only capture the average human so far, and do not take individual differences into account; the definition of "normal" thus brings ethical questions with it, as it could amplify existing biases.

Second, the field of computer vision may benefit from a reduced number of weight updates which reduces training time, and we hope this will make the resulting models more accessible to researchers without access to large compute resources. These models have so far not been thoroughly tested on a range of benchmarks other than ImageNet and generalization to other classification tasks is therefore unproven.

\section{Acknowledgments and Disclosure of Funding}

We thank Corey Ziemba and Anthony Movshon for access to the V1 and V2 data, Magdalena Geiger as well as Michiel Haisma for support in figure designs and Dean Pospisil for helpful discussions. This work was supported the Prosa scholarship of LMU, foundation of the University of Augsburg, the Foundation "Rohde Stiftung" (F.G.), the Massachusetts Institute of Technology Shoemaker Fellowship (M.S.), the SRC Semiconductor Research Corporation and DARPA (M.S., J.J.D.), the PhRMA Foundation Postdoctoral Fellowship in Informatics (T.M.), and Simons Foundation grant SCGB-542965 (J.J.D.).

\section{References}

[1] Martin Schrimpf, Jonas Kubilius, Ha Hong, Najib J. Majaj, Rishi Rajalingham, Elias B. Issa, Kohitij Kar, Pouya Bashivan, Jonathan Prescott-Roy, Kailyn Schmidt, Daniel L. K. Yamins, and James J. DiCarlo. Brain-Score: Which artificial neural network for object recognition is most brain-like? bioRxiv, 2018.

[2] Jonas Kubilius, Martin Schrimpf, Ha Hong, Najib J. Majaj, Rishi Rajalingham, Elias B. Issa, Kohitij Kar, Pouya Bashivan, Jonathan Prescott-Roy, Kailyn Schmidt, Aran Nayebi, Daniel Bear, Daniel L. K. Yamins, and James J. DiCarlo. Brain-Like Object Recognition with High-Performing Shallow Recurrent ANNs. In Neural Information Processing Systems (NeurIPS), pp. 12785-12796. 2019.

[3] Jia Deng, Wei Dong, Richard Socher, Li-Jia Li, Kai Li, and Li Fei-Fei. ImageNet: A large-scale hierarchical image database. In IEEE Conference on Computer Vision and Pattern Recognition, pp. 248-255. 2009.

[4] Daniel LK Yamins, Ha Hong, Charles F Cadieu, Ethan A Solomon, Darren Seibert, and James J DiCarlo. Performance-optimized hierarchical models predict neural responses in higher visual cortex. Proceedings of the National Academy of Sciences, 111(23):8619-8624, 2014.

[5] Seyed-Mahdi Khaligh-Razavi and Nikolaus Kriegeskorte. Deep supervised, but not unsupervised, models may explain it cortical representation. PLoS computational biology, 10(11):e1003915, 2014.

[6] Santiago A Cadena, George H Denfield, Edgar Y Walker, Leon A Gatys, Andreas S Tolias, Matthias Bethge, and Alexander S Ecker. Deep convolutional models improve predictions of macaque v1 responses to natural images. bioRxiv, pp. 201764, 2017.

[7] Hanlin Tang, Martin Schrimpf, William Lotter, Charlotte Moerman, Ana Paredes, J.O. Josue Ortega Caro, Walter Hardesty, David Cox, and Gabriel Kreiman. Recurrent computations for visual pattern completion. Proceedings of the National Academy of Sciences (PNAS), 115(35):8835-8840, 2018.

[8] Rishi Rajalingham, Elias B Issa, Pouya Bashivan, Kohitij Kar, Kailyn Schmidt, and James J DiCarlo. Large-scale, high-resolution comparison of the core visual object recognition behavior of humans, monkeys, and state-of-the-art deep artificial neural networks. Journal of Neuroscience, pp. 7255-7269, 2018. 
[9] Anthony Zador. A Critique of Pure Learning: What Artificial Neural Networks can Learn from Animal Brains. bioRxiv preprint, 2019.

[10] J. Anthony Movshon and Lynne Kiorpes. Analysis of the development of spatial contrast sensitivity in monkey and human infants. Journal of the Optical Society of America A (JOSA A), 5(12):2166, 1988.

[11] Lynne Kiorpes and J. Anthony Movshon. Development of sensitivity to visual motion in macaque monkeys. Visual Neuroscience, 21(6):851-859, 2004.

[12] Darren Seibert. High-level visual object representation in juvenile and adult primates. PhD thesis, Massachusetts Institute of Technology, 2018.

[13] Geoffrey Hinton, Oriol Vinyals, and Jeff Dean. Distilling the Knowledge in a Neural Network. arXiv preprint, 2015.

[14] Jang Hyun Cho and Bharath Hariharan. On the efficacy of knowledge distillation. In International Conference on Computer Vision (ICCV), volume 2019-Octob, pp. 4793-4801. 2019.

[15] Yonglong Tian, Dilip Krishnan, and Phillip Isola. Contrastive Representation Distillation. arXiv preprint, 2019.

[16] Nicholas Cheney, Martin Schrimpf, and Gabriel Kreiman. On the Robustness of Convolutional Neural Networks to Internal Architecture and Weight Perturbations. arXiv preprint, 2017.

[17] Ari S. Morcos, David G. T. Barrett, Neil C. Rabinowitz, and Matthew Botvinick. On the importance of single directions for generalization. In International Conference on Learning Representations (ICLR), 2018.

[18] Yonglong Tian, Yue Wang, Dilip Krishnan, Joshua B. Tenenbaum, and Phillip Isola. Rethinking Few-Shot Image Classification: a Good Embedding Is All You Need? arXiv preprint, 2020.

[19] Jonathan Frankle, David J. Schwab, and Ari S. Morcos. Training BatchNorm and Only BatchNorm: On the Expressive Power of Random Features in CNNs. arXiv preprint, 2020.

[20] Jonathan Frankle, Gintare Karolina Dziugaite, Daniel M. Roy, and Michael Carbin. The Lottery Ticket Hypothesis at Scale. arXiv preprint, 2019.

[21] Vivek Ramanujan, Mitchell Wortsman, Aniruddha Kembhavi, Ali Farhadi, and Mohammad Rastegari. What's Hidden in a Randomly Weighted Neural Network? arXiv preprint, 2019.

[22] Mathilde Caron, Piotr Bojanowski, Armand Joulin, and Matthijs Douze. Deep Clustering for Unsupervised Learning of Visual Features. In European Conference on Computer Vision (ECCV), pp. 132-149, 2018.

[23] Zhirong Wu, Yuanjun Xiong, Stella X Yu, and Dahua Lin. Unsupervised Feature Learning via Nonparametric Instance Discrimination. In Computer Vision and Pattern Recognition (CVPR), pp. 3733-3742, 2018.

[24] Chengxu Zhuang, Alex Zhai, and Daniel Yamins. Local aggregation for unsupervised learning of visual embeddings. In International Conference on Computer Vision (ICCV), pp. 6001-6011. 2019.

[25] Olivier J. Hénaff, Aravind Srinivas, Jeffrey De Fauw, Ali Razavi, Carl Doersch, S. M. Ali Eslami, and Aaron van den Oord. Data-Efficient Image Recognition with Contrastive Predictive Coding. In Computer Vision and Pattern Recognition (CVPR), 2019.

[26] Jeremy Freeman, Corey M Ziemba, David J Heeger, Eero P Simoncelli, and J Anthony Movshon. A functional and perceptual signature of the second visual area in primates. Nature Neuroscience, 16(7): 974-981, 2013.

[27] Najib J Majaj, Ha Hong, Ethan A Solomon, and James J DiCarlo. Simple learned weighted sums of inferior temporal neuronal firing rates accurately predict human core object recognition performance. Journal of Neuroscience, 35(39):13402-13418, 2015.

[28] Kaiming He, Xiangyu Zhang, Shaoqing Ren, and Jian Sun. Delving Deep into Rectifiers: Surpassing Human-Level Performance on ImageNet Classification. In Proceedings of the IEEE international conference on computer vision, pp. 1026-1034, 2015.

[29] G Leuba and R Kraftsik. Changes in volume, surface estimate, three-dimensional shape and total number of neurons of the human primary visual cortex from midgestation until old age. Anatomy and Embryology, 190(4):351-366, 1994.

[30] Evelyn Fix and J. L. Hodges. Discriminatory analysis, nonparametric discrimination. Technical report, United States Air Force, 1951.

[31] Robert L. Thorndike. Who belongs in the family? Psychometrika, 18(4):267-276, 1953.

[32] David H Hubel and Torsten N Wiesel. Receptive fields, binocular interaction and functional architecture in the cat's visual cortex. The Journal of physiology, 160(1):106-154, 1962.

[33] J. P. Jones and L. A. Palmer. The two-dimensional spatial structure of simple receptive fields in cat striate cortex. Journal of Neurophysiology, 58(6):1187-1211, 1987.

[34] Andrew D. Huberman, Marla B. Feller, and Barbara Chapman. Mechanisms Underlying Development of Visual Maps and Receptive Fields. Annual Review of Neuroscience, 31(1):479-509, 2008. 
[35] Andrew G. Howard, Menglong Zhu, Bo Chen, Dmitry Kalenichenko, Weijun Wang, Tobias Weyand, Marco Andreetto, and Hartwig Adam. MobileNets: Efficient Convolutional Neural Networks for Mobile Vision Applications. arXiv preprint, 2017.

[36] Kaiming He, Xiangyu Zhang, Shaoqing Ren, and Jian Sun. Deep residual learning for image recognition. In Proceedings of the IEEE conference on computer vision and pattern recognition, pp. 770-778, 2016.

[37] Maximilian Riesenhuber and Tomaso Poggio. Hierarchical models of object recognition in cortex. Nature neuroscience, 2(11):1019, 1999.

[38] Matthew D Zeiler and Rob Fergus. Visualizing and Understanding Convolutional Networks. arXiv preprint, 2013.

[39] Chris Olah, Nick Cammarata, Ludwig Schubert, Gabriel Goh, Michael Petrov, and Shan Carter. Zoom In: An Introduction to Circuits. Distill, 5(3), 2020.

[40] Nick Cammarata, Shan Carter, Gabriel Goh, Chris Olah, Michael Petrov, and Ludwig Schubert. Thread: Circuits. Distill, 2020. https://distill.pub/2020/circuits.

[41] Stephen Grossberg. Competitive learning: From interactive activation to adaptive resonance. Cognitive Science, 11(1):23-63, 1987.

[42] James C.R. Whittington and Rafal Bogacz. Theories of Error Back-Propagation in the Brain. Trends in Cognitive Sciences, 23(3):235-250, 2019.

[43] Eric Hunsberger. Spiking Deep Neural Networks: Engineered and Biological Approaches to Object Recognition. PhD thesis, University of Waterloo, 2017.

[44] Gideon Schwarz. Estimating the Dimension of a Model. Annals of Statistics, 6(2):461-464, 1978.

\section{A Weight compression details}

\section{A.1 Compressing the first layer with a Gabor prior}

The weight compression approach we use in Section 4 is based on different initialization techniques, applied to different layers. For the very first layer of size $7 \times 7$ we found a Gabor filter most effective. To generate the Gabor kernels we fit trained channel weights to a Gabor function

$$
G_{\theta, f, \phi, n_{x}, n_{y}, C}(x, y)=\frac{1}{2 \pi \sigma_{x} \sigma_{y}} \exp \left[-0.5\left(x_{r o t}^{2} / \sigma_{x}^{2}+y_{r o t}^{2} / \sigma_{y}^{2}\right)\right] \cos (2 \pi f+\phi) C
$$

where

$$
\begin{aligned}
& x_{\text {rot }}=x \cos (\theta)+y \sin (\theta) \\
& y_{\text {rot }}=-x \sin (\theta)+y \cos (\theta)
\end{aligned}
$$

$$
\begin{gathered}
\sigma_{x}=\frac{n_{x}}{f} \\
\sigma_{y}=\frac{n_{y}}{f}
\end{gathered}
$$

$x_{r o t}$ and $y_{r o t}$ are the orthogonal and parallel orientations relative to the grating, $\theta$ is the angle of the grating orientation, $f$ is the spatial frequency of the grating, $\phi$ is the phase of the grating relative to the Gaussian envelope, $\sigma_{x}$ and $\sigma_{y}$ are the standard deviations of the Gaussian envelope orthogonal and parallel to the grating, which can be defined as multiples $\left(n_{x}\right.$ and $\left.n_{y}\right)$ of the inverse of the grating frequency and $C$ is a scaling factor.

The function is fit per channel, which leads to a set of Gabor parameter for each of the 3 RGB channels. We then fit a multidimensional mixture of Gaussians to the combination of all filter parameter per kernel, resulting in a kernel parameter set. For the three RGB input channels in the first layer and the 8 Gabor parameters we therefore fit to $3 \times 8=27$ parameters. We evaluate the best number of components (number of distinct Gaussian distributions) based on the Bayesian Information Criterion [44]. To generate new kernels we sample a kernel parameter set from this mixture distribution and apply them to the described Gabor function that spans the weight values.

\section{A.2 Compressing BatchNorm layers}

In addition to convolutional layers, models consist of several Batchnorm layers, which contain a learnable bias and weight term. To initialize these terms, we fit a normal distribution per weight and bias vector of the trained values and sample from this distribution. Note that BatchNorm layers contain running average means and standard deviations for normalization purposes. Those terms are set to zero when no training has happened. During training the mean and standard deviation of 


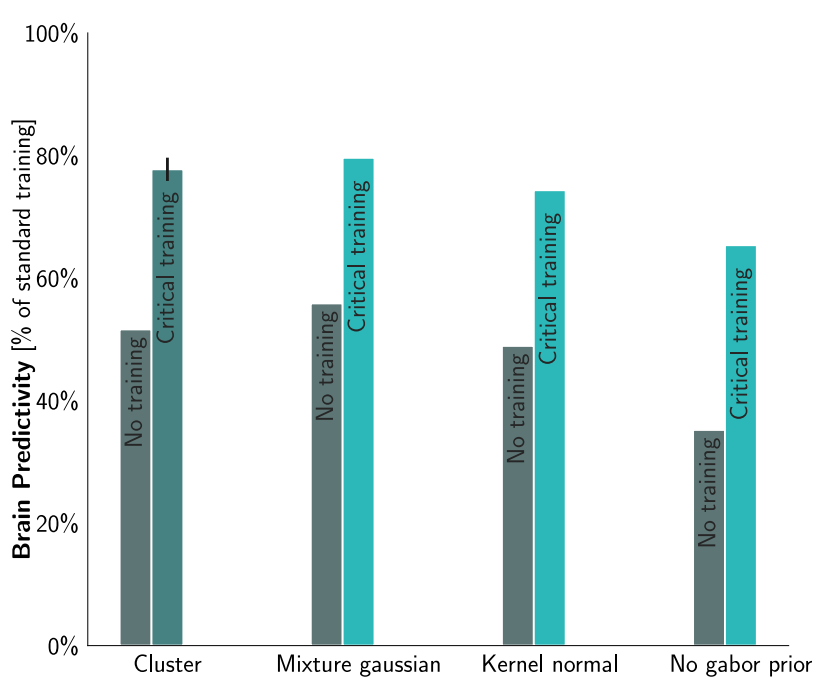

Figure 6: Alternative weight compression methods Comparison of different initializations that compress weights, "at birth" i.e. without any training (gray) and after training critical layers (shades of blue) for 6 epochs. Our best clustering-based approach $W C$ achieved similar results as the Mixture Gaussian approach ( $\sim 3$ percent points mean difference) but leads to more diverse clusters. Performance drops when solely sampling weights from kernel based normal distributions (Kernel normal) and additionally disabling the Gabor prior (No Gabor prior)

the current batch are used instead. At validation time to achieve consistent results over epochs, we disable updates of running mean and averages and set them to a trained models values.

\section{A.3 Alternative approaches}

We have explored a variety of weight compression methods applied to different layers and evaluate their performance "at birth" without training and when trained with critical training.

Figure 6 shows brain predictivities of several alternative compression methods implemented as follows:

- WC Weight compression approach with clustering as described in Section 4, using a Gabor prior approach for the first layer, noisy cluster sampling for convolutional layers and fitted normal distributions for Batchnorm layers.

- Mixture Gaussian Instead of sampling weights from cluster centers, this approach uses multidimensional distributions for convolutional layers with kernel size $>1$. We fit a mixture Gaussian distribution per layer to the weights of a channel over all kernels. To sample a new kernel, we sample individual channels from this distribution. For convolutional layers with kernel size $==1$ we draw weights from a normal distribution adjusted per kernel as described in the next item.

- Kernel normal All weights are sampled based on normal distributions. We fit mean and standard deviation to the weights of one trained kernel and resample a new kernel from this distribution. We do this separately for every kernel to generate a whole layer. This approach is similar to the BatchNorm sampling method where we compress BatchNorm weight and bias terms instead of kernels.

- No Gabor prior To evaluate the importance of the Gabor prior we use the Kernel normal model and apply the same normal distribution approach to layer one instead of Gabor sampling. Performance drops by 13 percent points without training, and by 9 percent points after critical training.

\section{B WC initialized and $C T$ trained model analysis}

Our best model $W C+C T$ benefits from a combination of improved initialization through weight compression, and critical training. Figure 7A shows models with standard initialization and training all weights, but with fewer supervised updates (cf. Figure 1), models that only train down-sampling layers $(C T)$, and models that combine critical training with weight compression $(W C+C T)$. A model initialized with weight compression achieves (only $W C$ ) $54 \%$ brain predictivity with 0 supervised synaptic updates. Figure 7B and $\mathrm{C}$ show detailed brain predicitivity scores, relative to a fully trained 

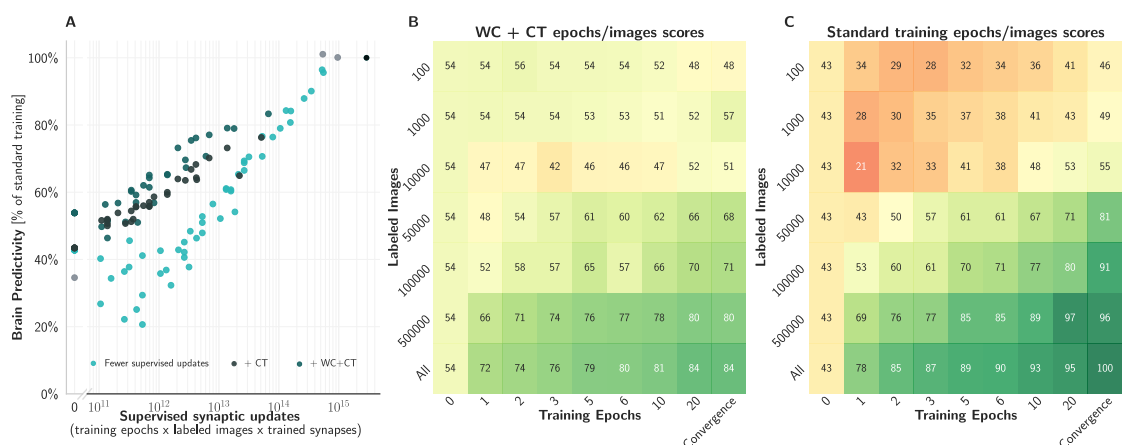

Figure 7: Detailed analysis of $\boldsymbol{W C}+\boldsymbol{C T}$. A When reducing the number of supervised synaptic updates, adding critical training (dark grey) and adding weight compression initialization (dark blue) both improve brain predictivity at the same number of supervised synaptic updates, in comparison to a model with standard initialization and training all weights (bright blue). B Brain predictivities for the $W C+C T$ model when trained with a range of epochs and labeled images. C Same as B, but for a standardly initialized $(K N)$ model training all weights.
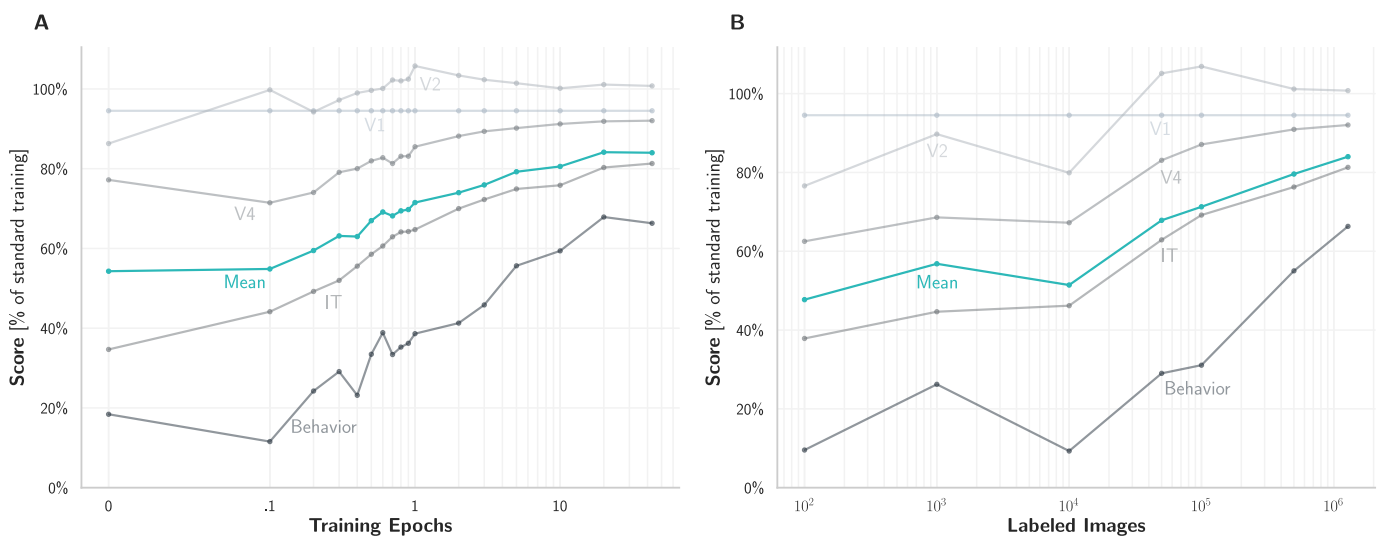

Figure 8: Individual brain benchmark scores for $\boldsymbol{W C}+\boldsymbol{C T}$ model A Individual brain predictivities of $W C+C T$ models trained with a range of epochs on all images. These models score especially high on V1, V2 and V4 already after one epoch in comparison to a model with standard initialization training all weights. IT and Behavior benchmarks continuously improve over later epochs as well but fall short of a fully trained model. B Like A, but with models trained until convergence on different numbers of labeled images, up to the full dataset of $1.28 \mathrm{M}$ images (rightmost points). As in A we see $>80 \%$ V1, V2 and V4 scores with only 100,000 images. For comparable IT and behavioral scores, more images are required.

model, for models initialized and trained with $W C+C T$ (B) and models initialized with standard Kaiming Normal and training all weights $(C)$ when trained with a range of epochs and labeled images. The specific benchmark scores when either training with all labeled images for a varying number of epochs (Figure 8A) or when training with fewer labeled images until convergence (Figure 8B) show the benchmarks of early visual achieve the best results, relative to a fully trained model. The V1 score is identical over all training states, since we do not train the V1 area.

\section{Dissecting training reductions - details}

\section{C.1 Transfer to ResNet and MobileNet}

To show the generalization of our approach we applied the weight compression methods to a ResNet50 [36] and a MobileNet [35] (version 1, multiplier 1.0, image size 224) architecture. We do not regenerate sampling distributions or clusters based on the new architectures trained weights, but 
used the CORnet-S based distributions to sample new weights for the different architectures. Since CORnet-S is inspired by ResNet modules, we applied our critical training approach by training all conv3 layers (equivalent down sampling layers) of ResNet50. For MobileNet we explored various layer mappings. When training only the very few layers that result in reduced feature size, which are implemented as depthwise separable convolutional layers and appear three times overall, performance performance dropped close to random. Those layers however are mapped to CORnet-S' conv2 layers due to their $3 \times 3$ kernels whereas critical training in CORnet-S trains conv3 down-sampling layers with a kernel size of $1 \times 1$. To transfer our critical training approach, we therefore additionally train the $1 \times 1$ MobileNet layers corresponding to conv 3 . This training version allows for more training but still reduces the amount of trained parameters by $43 \%$ while maintaining $78 \%$ of the original score. For both transfer methods we initialize the first layer using the Gabor method based on CORnet-S's mixture-of-Gaussian distribution. Since the Gabor function is scalable we can produce Gabor kernels of varying size. Furthermore we disable BatchNorm biases and weights in all transfer models by freezing them to default values. We found that transferring those distributions on new architectures harms brain predictivity scores. Nevertheless, the BatchNorm layers still normalize activations by applying the running average and standard deviation.

\section{C.2 Comparison of techniques to reduce supervised synaptic updates (Fig. 5B)}

To analyse the relative contributions of Weight Compression and Critical Training we compare brain predictivities of different models in Figure 5B:

- KN A model initialized by standard Kaiming Normal initialization without training.

- WC A model initialized by our Weight Compression initialization, described in Section 4, without training.

- $\mathrm{KN}+\mathrm{CT}$ The $K N$-initialized model trained with Critical Training until convergence, i.e. three downstream layers and the decoder are trained and all other layers remain unchanged.

- WC+CT The $W C$-initialized model with Critical Training. V1 scores do not change because weights in the V1 model area are all frozen.

\section{Training details}

We used PyTorch 0.4.1 and trained the model using the ImageNet 2012 training set [3]. We used a batch size of 256 images and trained on a QuadroRTX6000 GPU until convergence. We start with a learning rate of 0.1 and decrease it four times by a factor of ten when training loss does not decrease over a period of three epochs. For optimization, we use Stochastic Gradient Descent with a weight decay 0.0001 , momentum 0.9 , and a cross-entropy loss between image labels and model logits. We trained all models with these settings except the standard Mobilenet, where we used the pretrained tensorflow model. Since the number of epochs for this model are not clearly stated, we use the published value of 100 training epochs [35]. The training time of a full CORnet-S with standard Imagenet dataset for 43 epochs is $\sim 2.5$ days. All variations with less weights/images/epochs trained in shorter time. Reference models trained for 4 days at most under the described settings. If not further specified, we show results of one training run. When showing error bars we used seeds 0 and 42 or when $n=3$ we use seeds 0,42 and 94 .

Code to reproduce our analyses from scratch, including the framework for weight compression and critical training, as well as pre-trained models, will be made available through GitHub. 